\title{
THE STONE-ČECH COMPACTIFICATION, THE STONE-ČECH REMAINDER, AND THE REGULAR WALLMAN PROPERTY
}

\author{
TAKASHI KIMURA
}

\begin{abstract}
In this paper, the following are proved: (1) the Stone-Čech compactification of a metrizable space is regular Wallman, (2) if the Stone-Čech compactification of a locally compact space whose pseudocompact closed subsets are compact is regular Wallman, then the Stone-Čech remainder is also regular Wallman. Consequently, the Stone-Cech remainder of a locally compact metrizable space is regular Wallman.
\end{abstract}

1. Introduction. Throughout this paper, by a space we mean a completely regular $T_{1}$-space.

A compactification which arises from a normal base is called Wallman. A compact space is regular Wallman if it has a normal base consisting of regular closed sets. Every regular Wallman space is a Wallman compactification of each of its dense subspaces [10].

The Stone-Čech compactification $\beta X$ of a space $X$ is always Wallman [6], however, it need not be regular Wallman (see Example 3.8). Misra [8] proved that $\beta X$ is regular Wallman in case $X$ is separable and metrizable. Van Mill [7] extended this result to the case $X$ is strongly $\aleph_{1}$ compact. A space $X$ is strongly $\aleph_{1}$ compact if for each subset $A$ of $X$ with $|A| \geqslant \aleph_{1}$ and for each total order $<$ on $A$ there is $y \in A$ such that for each neighborhood $U$ of $y$ both $U \cap\{a \in A \mid a<y\}$ and $U \cap\{a \in A \mid y<a\}$ are nonempty. Strongly $\aleph_{1}$ compact spaces are known to be hereditarily Lindelöf and hereditarily separable [1]. Therefore, the question of whether Misra's result above can be true for nonseparable case arises naturally. In $\S 3$ we shall prove that $\beta X$ is regular Wallman for an arbitrary metrizable space $X$.

It is known that the regular Wallman property is not closed-hereditary ([9] or [11]). In particular, for a locally compact space $X$ it is not known whether the Stone-Čech remainder $X^{*}=\beta X \backslash X$ is regular Wallman even if $\beta X$ is regular Wallman. In $\S 4$ we shall prove that $X^{*}$ is regular Wallman in case $X$ is locally compact and metrizable.

\section{Preliminaries.}

2.1. Definitions. Let $\mathscr{S}$ and $\mathscr{T}$ be collections of subsets of a space $X$. We shall write $\cap \mathscr{S}$ for $\cap\{S \mid S \in \mathscr{S}\}, \cup \mathscr{S}$ for $\cup\{S \mid S \in \mathscr{S}\}, \mathrm{C} 1 \mathscr{S}$ for $\{\mathrm{C} 1 S \mid S \in \mathscr{S}\}$ and

Received by the editors March 25, 1985 and, in revised form, July 15, 1985 and December 30, 1985. 1980 Mathematics Subject Classification. Primary 54D35, 54D40.

Key words and phrases. Regular Wallman, Stone-Čech compactification, Stone-Čech remainder. 
$\wedge . \mathscr{S}$ for $\left\{\cap \mathscr{S}^{\prime} \mid \mathscr{S}^{\prime}\right.$ is a finite subcollection of $\left.\mathscr{S}\right\} . \mathscr{S}$ separates $\mathscr{T}$ if for $T_{0}, T_{1} \in \mathscr{T}$ with $T_{0} \cap T_{1}=\varnothing$ there are $S_{0}, S_{1} \in \mathscr{S}$ such that $T_{0} \subset S_{0}, T_{1} \subset S_{1}$, and $S_{0} \cap S_{1}=\varnothing . \mathscr{S}$ separates disjoint closed subsets of $X$ if $\mathscr{S}$ separates the collection of all closed subsets of $X . \mathscr{S}$ is closure-distributive if for each finite subcollection $\mathscr{S}^{\prime}$ of $\mathscr{S}$ it is true that $\cap \mathrm{Cl} \mathscr{S}^{\prime}=\mathrm{Cl}\left(\cap \mathscr{S}^{\prime}\right) . \mathscr{S}$ is a ring if $\mathscr{S}$ is closed under finite unions and finite intersections. $\mathscr{S}$ is separating if $F$ is closed in $X$ and $x \in X \backslash F$ implies that there are $S_{0}, S_{1} \in \mathscr{S}$ such that $F \subset S_{0}, x \in S_{1}$ and $S_{0} \cap S_{1}=\varnothing . \mathscr{S}$ is normal if for $T_{0}, T_{1} \in \mathscr{T}$ with $T_{0} \cap T_{1}=\varnothing$ there are $S_{1}, S_{1} \in \mathscr{S}$ such that $S_{0} \cap T_{1}=\varnothing, S_{1} \cap T_{0}=\varnothing$ and $S_{0} \cup S_{1}=X . \mathscr{S}$ is a normal base for $X$ if $\mathscr{S}$ is a normal, separating ring consisting of closed subsets of $X$. For each normal base $\mathscr{F}$ for a space $X$ we denote by $w(X, \mathscr{F})$ the Wallman space of $X$ with respect to $\mathscr{F}$. It is well known that $w(X, \mathscr{F})$ is a compactification of $X$. For two compactifications $\alpha X$ and $\gamma X$ of a space $X$ we say that $\alpha X \geqslant \gamma X$ if there is a continuous mapping $f$ : $\alpha X \rightarrow \gamma X$ with $f_{\mid X}=\operatorname{id}_{X}$.

The following two lemmas were proved by Steiner [10].

2.2. Lemma. Let $\mathscr{F}$ be a normal base for a space $X$ and $\alpha X=w(X, \mathscr{F})$. Then $\mathrm{Cl}_{\alpha X} \mathscr{F}$ is a normal base for $\alpha X$.

2.3. Lemma. Let $\mathscr{S}$ and $\mathscr{T}$ be normal bases for a space $X$. Then $w(X, \mathscr{S}) \geqslant w(X, \mathscr{T})$ if and only if $\mathscr{S}$ separates $\mathscr{T}$.

2.4. RemarK. By Lemma 2.3, if a normal base $\mathscr{F}$ for a normal space $X$ separates disjoint closed subsets of $X$, then we have $w(X, \mathscr{F})=\beta X$.

3. The Stone-Čech compactification. The main result of this section is as follows.

3.1. THEOREM. The Stone-Čech compactification of a metrizable space is regular Wallman.

This is a generalization of a result of Misra [8]. The idea of the proof of Theorem 3.1 is essentially due to Misra. Indeed, we make use of the following lemmas.

3.2. Lemma (MisRa [8]). Let $X$ be a normal space and $\left\{\left(A_{n}, C_{n}\right) \mid n \in \mathbf{N}\right\} a$ collection of pairs of open subsets of $X$ with $\mathrm{Cl} A_{n} \subset C_{n}$. Then there is a closure-distributive collection $\left\{B_{n} \mid n \in \mathbf{N}\right\}$ of open subsets of $X$ such that $\mathrm{Cl} A_{n} \subset B_{n} \subset \mathrm{Cl} B_{n} \subset C_{n}$ for each $n \in \mathbf{N}$.

3.3. Lemma (MiSRa [8]). Let $\mathscr{A}$ be a closure-distributive collection of subsets of a space $X$. Let

$$
\mathscr{A}^{*}=\{\bigcup \mathscr{B} \mid \mathscr{B} \subset \Lambda . \mathscr{A} \text { and } \mathrm{Cl}(\bigcup \mathscr{B})=\bigcup \mathrm{Cl} \mathscr{B}\}
$$

Then $\mathscr{A}^{*}$ is a closure-distributive ring.

3.4. Lemma. For each $n \in \mathbf{N}$ let $\mathscr{U}_{n}=\left\{U_{\alpha} \mid \alpha \in \Lambda_{n}\right\}$ and $\mathscr{V}_{n}=\left\{V_{\alpha} \mid \alpha \in \Lambda_{n}\right\}$ be discrete collections of open subsets of a normal space $X$ with $\mathrm{Cl} U_{\alpha} \subset V_{\alpha}$. Then there is a collection $\mathscr{W}_{n}=\left\{W_{\alpha} \mid \alpha \in \Lambda_{n}\right\}$ of open subsets of $X$ for each $n \in \mathbf{N}$ such that 
$\mathrm{Cl} U_{\alpha} \subset W_{\alpha} \subset \mathrm{Cl} W_{\alpha} \subset V_{\alpha}$ for each $\alpha \in \Lambda_{n}$ and each $n \in \mathbf{N}$ and $\mathscr{W}=\bigcup\left\{\mathscr{W}_{n} \mid n \in\right.$ $\mathbf{N}$ \} is closure-distributive.

Proof. As $X$ is normal, for each $\alpha \in \Lambda_{n}$ and each $n \in \mathbf{N}$ there is an open subset $O_{\alpha}$ of $X$ with $\mathrm{Cl} U_{\alpha} \subset O_{\alpha} \subset \mathrm{Cl} O_{\alpha} \subset V_{\alpha}$. Let $U_{n}=\bigcup \mathscr{U}_{n}$ and $O_{n}=\bigcup\left\{O_{\alpha} \mid \alpha \in \Lambda_{n}\right\}$ for each $n \in \mathbf{N}$. As $\mathscr{U}_{n}$ is discrete, we have $\mathrm{Cl} U_{n} \subset O_{n}$. By Lemma 3.2, there is a closure-distributive collection $\left\{W_{n} \mid n \in \mathbf{N}\right\}$ of open subsets of $X$ such that $\mathrm{Cl} U_{n} \subset$ $W_{n} \subset \mathrm{Cl} W_{n} \subset O_{n}$ for each $n \in \mathbf{N}$. Let $W_{\alpha}=O_{\alpha} \cap W_{n}$ for each $\alpha \in \Lambda_{n}$ and each $n \in \mathbf{N}$, and $\mathscr{W}_{n}=\left\{W_{\alpha} \mid \alpha \in \Lambda_{n}\right\}$. Then $\mathscr{W}_{n}$ is discrete, because $W_{\alpha} \subset V_{\alpha}$ for each $\alpha \in \Lambda_{n}$ and $\mathscr{V}_{n}$ is discrete. To see that $\mathscr{W}=\bigcup\left\{\mathscr{W}_{n} \mid n \in \mathbf{N}\right\}$ is closure-distributive, let $W_{\alpha(i)} \in \mathscr{W}_{n(i)}$ and $x \in \bigcap\left\{\mathrm{Cl} W_{\alpha(i)} \mid i \leqslant m\right\}$. We show that $x \in \operatorname{Cl}\left(\cap\left\{W_{\alpha(i)} \mid i \leqslant\right.\right.$ $m\})$. Let $\mathscr{A}$ be the collection of all sets of the form $\cap\left\{W_{\beta(i)} \mid i \leqslant m\right\}$, where $W_{\beta(i)} \in \mathscr{W}_{n(i)}$ for each $i$. Since $\mathscr{W}_{n(i)}$ is discrete for each $i, \mathscr{A}$ is discrete. Since

$$
\begin{aligned}
X & \in \bigcap\left\{\mathrm{Cl} W_{\alpha(i)} \mid i \leqslant m\right\} \subset \mathrm{Cl}\left(\bigcap\left\{W_{n(i)} \mid i \leqslant m\right\}\right) \\
& =\mathrm{Cl}(\bigcup \mathscr{A})=\bigcup \mathrm{Cl} \mathscr{A},
\end{aligned}
$$

we can take $\beta(i) \in \Lambda_{n(i)}$ for each $i$ so that $x \in \operatorname{Cl}\left(\cap\left\{W_{\beta(i)} \mid i \leqslant m\right\}\right)$. Then for each $i$ we have $\alpha(i)=\beta(i)$ and so $x \in \mathrm{Cl}\left(\cap\left\{W_{\alpha(i)} \mid i \leqslant m\right\}\right)$, since $x \in \mathrm{Cl} W_{\alpha(i)} \cap \mathrm{Cl} W_{\beta(i)}$ and $\mathscr{W}_{n(i)}$ is discrete. This completes the proof of Lemma 3.4.

3.5. LEMMA. If $X$ is dense in $Y$ and $F$ is a regular closed subset of $X$, then $\mathrm{Cl}_{Y} F$ is regular closed in $Y$.

3.6. Proof of Theorem 3.1. Let $X$ be a metrizable space. By $[5,4.4 .1]$, there is an open cover $\mathscr{B}_{n}=\bigcup\left\{\mathscr{B}_{n m} \mid m \in \mathbf{N}\right\}$ of $X$ such that

(1) $\mathscr{B}_{n}$ is locally finite,

(2) $\mathscr{B}_{n m}=\left\{B_{\alpha} \mid \alpha \in \Lambda_{n m}\right\}$ is discrete, and

(3) $\operatorname{diam} B<1 / n$ for each $B \in \mathscr{B}_{n}$.

Since $X$ is metrizable, there is an open shrinking $\mathscr{B}_{n}^{\prime}=\bigcup\left\{\mathscr{B}_{n m}^{\prime} \mid m \in \mathbf{N}\right\}$ of $\mathscr{B}_{n}$, where $\mathscr{B}_{n m}^{\prime}=\left\{B_{\alpha}^{\prime} \mid \alpha \in \Lambda_{n m}\right\}$. Then, by (2) and Lemma 3.4, for each $n, m \in \mathbf{N}$ there is a collection $\mathscr{U}_{n m}=\left\{U_{\alpha} \mid \alpha \in \Lambda_{n m}\right\}$ of open subsets of $X$ such that

(4) $\mathrm{Cl} B_{\alpha}^{\prime} \subset U_{\alpha} \subset \mathrm{Cl} U_{\alpha} \subset B_{\alpha}$ for each $\alpha \in \Lambda_{n m}$, and

(5) $\mathscr{U}=\bigcup\left\{\mathscr{U}_{n m} \mid n, m \in \mathbf{N}\right\}$ is closure-distributive.

Let $\mathscr{U}_{n}=\bigcup\left\{\mathscr{U}_{n m} \mid m \in \mathbf{N}\right\}$ for each $n \in \mathbf{N}$. Then by (1), (3) and (4), $\mathscr{U}_{n}$ is a locally finite open cover of $X$ and $\operatorname{diam} U<1 / n$ for each $U \in \mathscr{U}_{n}$. Thus $\mathscr{U}$ is a base for the open subsets of $X$. Let

$$
\mathscr{U}^{*}=\{\bigcup \mathscr{V} \mid \mathscr{V} \subset \Lambda \cdot \mathscr{U} \text { and } \mathrm{Cl}(\bigcup \mathscr{V})=\bigcup \mathrm{Cl} \mathscr{V}\}
$$

By (5) and Lemma 3.3, $\mathscr{U}^{*}$ is a closure-distributive ring for $X$. We shall prove that $\mathrm{Cl} \mathscr{U}^{*}$ separates disjoint closed subsets of $X$. To this end, let $F$ be a closed subset of $X$ and $U$ an open subset of $X$ containing $F$. For each $x \in F$ we take $U_{n} \in \mathscr{U}$ with $x \in U_{x} \subset \mathrm{Cl} U_{x} \subset U$ and set $\mathscr{V}=\left\{U_{x} \mid x \in F\right\}$. Obviously, $\mathscr{V} \subset \wedge$. $\mathscr{U}$. To see that $\mathrm{Cl}(\cup \mathscr{V})=\cup \mathrm{Cl} \mathscr{V}$, take $x \in \mathrm{Cl}(\cup \mathscr{V})$. If $x \in F$, then, obviously, $x \in \cup \mathrm{Cl} \mathscr{V}$. Suppose that $x \notin F$. Let $\delta=d(x, F)$, where $d$ is a compatible metric on $X, \mathscr{V}^{\prime}=$ $\bigcup\left\{\mathscr{U}_{n} \mid 2 / n>\delta\right\} \cap \mathscr{V}$. A routine calculation shows that $x \in \mathrm{Cl}\left(\cup \mathscr{V}^{\prime}\right)$. Since $\mathscr{V}^{\prime}$ 
is locally finite, we have $x \in \mathrm{Cl} V$ for some $V \in \mathscr{V}^{\prime}$. This implies that $\mathrm{Cl}(\mathrm{U} \mathscr{V})=$ $\cup \mathrm{Cl} \mathscr{V}$. Thus $\cup \mathscr{V} \in \mathscr{U}^{*}$. Obviously, $F \subset \cup \mathscr{V} \subset \mathrm{Cl}(\bigcup \mathscr{V}) \subset U$. Hence $\mathrm{Cl} \mathscr{U}^{*}$ separates disjoint closed subsets of $X$. It is easy to see that $\mathrm{Cl} \mathscr{U}^{*}$ is a normal base for $X$. By Remark 2.4, the Wallman space $w\left(X, \mathrm{Cl}_{\mathscr{U}^{*}}\right)$ is equivalent to $\beta X$. Let $\mathscr{F}=\mathrm{Cl}_{\beta X}\left(\mathrm{Cl}_{X} \mathscr{U}^{*}\right)$. Then, by Lemma $2.2, \mathscr{F}$ is a normal base for $\beta X$ and, by Lemma 3.5 , every member of $\mathscr{F}$ is regular closed in $\beta X$. Hence $\beta X$ is regular Wallman.

3.7. Lemma. Let $A$ be a closed subset of a regular Wallman space $X$. Then $X / A$ is also regular Wallman.

Proof. Suppose $\mathscr{S}$ is a normal base for $X$ consisting of regular closed subsets of $X$ and $f: X \rightarrow X / A$ is the quotient mapping. We set

$$
\mathscr{T}=\{f(S) \mid S \in \mathscr{S} \text { and either } S \cap A=\varnothing \text { or } A \subset S\} .
$$

Then $\mathscr{T}$ is a normal base for $X / A$ conisting of regular closed subsets of $X / A$. Hence $X / A$ is regular Wallman.

3.8. EXAMPLE. There is a noncompact space $X$ such that no compactification of $X$ is regular Wallman.

Let $Y$ be a compact space which is not regular Wallman, e.g. $Y$ is an example of Solomon [9] or an example of $\mathrm{Ul}^{\prime}$ janov [11]. Obviously, $Y$ has a nonisolated point $y$. Let $X=Y \backslash\{y\}$. Then the one-point compactification $\omega X=X \cup\{p\}$ is homeomorphic to $Y$. For each compactification $\alpha X$ of $X$ we have $\omega X=\alpha X / f^{-1}(p)$, where $f: \alpha X \rightarrow \omega X$ is a continuous mapping with $f_{\mid X}=\mathrm{id}_{X}$. Hence, by Lemma 3.7, $\alpha X$ is not regular Wallman. In particular, $\beta X$ is not regular Wallman.

3.9. Proposition. Let $A$ be a closed subset of a metrizable space $X$. Then the Stone-Čech compactification of $X / A$ is regular Wallman.

Proof. Since $\beta(X / A)=\beta X / \mathrm{Cl}_{\beta X} A$, it follows from Theorem 3.1 and Lemma 3.7.

Recall that a space $X$ is a Lašnev space if $X$ is the closed image of a metrizable space. In Proposition 3.9, obviously, $X / A$ is a Lašnev space.

3.10. QUESTION. Must the Stone-Čech compactification of a Lašnev space be regular Wallman?

4. The Stone-Čech remainder. We denote by $X^{*}$ the Stone-Čech remainder $\beta X \backslash X$ of a space $X$. Note that $X^{*}$ is compact if and only if $X$ is locally compact. Thus it is necessary that $X$ be locally compact in order that $X^{*}$ be regular Wallman. In this section we shall prove that $X^{*}$ is regular Wallman in certain cases. We need some lemmas.

4.1. Lemma. If $X$ is realcompact and $A$ is regular closed in $X$, then $\mathrm{Cl}_{\beta X} A \cap X^{*}$ is regular closed in $X^{*}$.

Proof. See $[12,2.9]$. 
4.2. LEMMA. If every pseudocompact closed subset of a space $X$ is compact, then $\beta X \backslash v X$ is dense in $X^{*}$, where $v X$ denotes the Hewitt realcompactification of $X$.

Proof. This follows from $[4,4.1]$.

4.3. THEOREM. Let $X$ be a locally compact space in which every pseudocompact closed subset is compact. If $\beta X$ is regular Wallman, then so is $X^{*}$.

Proof. Let $\mathscr{F}$ be a normal base for $\beta X$ consisting of regular closed subsets of $\beta X$. By Lemmas 3.5, 4.1 and 4.2, $F \cap X^{*}$ is regular closed in $X^{*}$ for every $F \in \mathscr{F}$. Thus $\left\{F \cap X^{*} \mid F \in \mathscr{F}\right\}$ is a normal base for $X^{*}$ consisting of regular closed subsets of $X^{*}$. Hence $X^{*}$ is regular Wallman.

The author wishes to thank the referee for strengthening Theorem 4.3 in an early version of this paper.

4.4. COROLLARY. The Stone-Čech remainder of a locally compact metrizable space is regular Wallman.

Proof. Apply Theorems 3.1 and 4.3.

4.5. RemarK. Van Mill [7] proved that the Stone-Čech compactification of a strongly $\boldsymbol{\aleph}_{1}$ compact space is regular Wallman. Since every strongly $\boldsymbol{\aleph}_{1}$ compact space is Lindelöf, the Stone-Čech remainder of a locally compact strongly $\boldsymbol{\aleph}_{1}$ compact space is regular Wallman. However, it is known that $X^{*}$ is an $F$-space if $X$ is locally compact and Lindelöf. Recall that a space $X$ is an $F$-space if disjoint cozero-sets of $X$ are completely separated. Biles [2] proved that every compact $F$-space is a $Z$-compactification (i.e. a Wallman compactification which arises from a normal base consisting of zero-sets) of each of its dense subspaces. This fact suggests that every compact $F$-space may be regular Wallman.

4.6. Proposition. Every compact F-space is regular Wallman.

Proof. Let $\mathscr{U}$ be the collection of all cozero-sets of the compact $F$-space $X$. Note that $\mathscr{U}$ is a base for the open subsets of $X$. We shall prove that $\mathscr{U}$ is closure-distributive. To see this it suffices to show that $\mathrm{Cl}\left(U_{0} \cap U_{1}\right)=\mathrm{Cl} U_{0} \cap \mathrm{Cl} U_{1}$ for $U_{0}$, $U_{1} \in \mathscr{U}$. Let $x \in \mathrm{Cl} U_{0} \cap \mathrm{Cl} U_{1}$. Then for each $V \in \mathscr{U}$ with $x \in V$ we have $x \in V$ $\cap \mathrm{Cl} U_{i} \subset \mathrm{Cl}\left(V \cap U_{i}\right), i=0,1$. As $X$ is an $F$-space, it follows that $V \cap U_{0} \cap U_{1} \neq$ $\varnothing$, therefore $x \in \mathrm{Cl}\left(U_{0} \cap U_{1}\right)$. It quickly follows that $\mathrm{Cl} \mathscr{U}$ is a normal base for $X$ consisting of regular closed subsets of $X$, and $X$ is regular Wallman.

4.7. CoROllaRY. The Stone-Čech remainder of a locally compact Lindelöf space is regular Wallman.

4.8. COROLlaRY. The Stone-Čech remainder of a locally compact F-space is regular Wallman.

4.9. EXAMPLE. There is a locally compact space $X$ such that the Stone-Čech remainder of $X$ is not regular Wallman.

Let $Y$ be a compact space which is not regular Wallman. Then there is a space $X$ with $X^{*}$ homeomorphic to $Y[3,4.17]$. Since $Y$ is compact, $X$ is locally compact. Obviously, $X^{*}$ is not regular Wallman. 


\section{REFERENCES}

1. E. S. Berney, Strongly $\boldsymbol{\aleph}_{1}$ compact spaces, Dissertation, Arizona State University, 1970.

2. C. M. Biles, Wallman-type compactifications, Proc. Amer. Math. Soc. 25 (1970), 363-369.

3. R. E. Chandler, Hausdorff compactifications, Lectures Notes in Pure and Appl. Math., vol. 23, Dekker, New York, 1976.

4. W. W. Comfort, On the Hewitt realcompactification of a product space, Trans. Amer. Math. Soc. 131 (1968), 107-118.

5. R. Engelking, General topology, PWN, Warsaw, 1977.

6. O. Frink, Compactifications and semi-normal spaces, Amer. J. Math. 86 (1964), 602-607.

7. J. van Mill, Compactifications in which the collection of multiple points is Lindelöf semi-stratifiable, Indag. Math. 38 (1976), 349-356.

8. A. K. Misra, Some regular Wallman $\beta X$, Indag. Math. 35 (1973), 237-242.

9. R. C. Solomon, A Hausdorff compactification that is not regular Wallman, General Topology and Appl. 7 (1977), 59-63.

10. E. F. Steiner, Wallman spaces and compactifications, Fund. Math. 61 (1968), 295-304.

11. V. M. Ul'janov, Solution of a basic problem on compactifications of Wallman type, Soviet Math. Dokl. 18 (1977), 567-571.

12. R. G. Woods, Co-absolutes of remainders of Stone-Čech compactifications, Pacific J. Math. 37 (1971), 545-560.

Institute of Mathematics, University of Tsukuba, Sakura-mura, Nilhari-gun, Ibaraki, 305 JAPAN 